\title{
Hemichorea associated with subcortical border-zone infarction and middle cerebral artery stenosis
}

\author{
So-Ei Ann, Suho Ro, Yun Hyeong Jeong, Sumin Kim, Pil-Wook Chung \\ Department of Neurology, Kangbuk Samsung Hospital, Sungkyunkwan University School of Medicine, Seoul, Korea
}

Received: September 8, 2021

Revised: November 25, 2021

Accepted: December 7, 2021

Corresponding author:

Pil-Wook Chung, MD, PhD

Department of Neurology,

Kangbuk Samsung Hospital,

Sungkyunkwan University School

of Medicine, 29 Saemunan-ro,

Jongno-gu, Seoul 03181, Korea

Tel: +82-2-2001-2050

Fax: +82-2-2001-2100

E-mail: pw.chung@samsung.com

\section{Dear Editor,}

Involuntary movement disorders sometimes occur after a stroke. One study reported that $1 \%$ of 2,500 stroke patients presented involuntary movement disorders [1]. These involuntary movement disorders include hypokinetic movement disorders and hyperkinetic movement disorders. Among these, hemichorea has been reported as the most common post-stroke hyperkinetic movement disorder $[1,2]$.

Post-stroke hemichorea is most commonly associated with a lesion in basal ganglia and related structures. In addition, several cases caused by cortical lesions have been reported. However, acute hemichorea associated with the lesions confined to subcortical white matter were very rarely reported $[2,3]$. We report a patient with subcortical border-zone infarction and middle cerebral artery (MCA) stenosis presenting with contralateral hemichorea.

A 79-year-old female presented with hyperkinetic movement of the left arm and leg, accompanied by dysarthria, which first occurred 10 days ago. She had no previous or family history and was not taking any medications.

At presentation, she appeared alert, and cooperative. Her muscle strength and sensory functions were normal. Deep-tendon reflexes were also normal and the Babinski sign was not elicited. There were involuntary, irregular and unpredictable muscle movements in her left arm and leg, consistent with chorea, more severe in the upper extremity. This involuntary movement was exaggerated during the intentional movement of the limb, and disappeared during sleep.

Laboratory tests, including blood sugar, uric acid, serum electrolytes, liver enzyme levels, and thyroid hormone levels, were all normal. She had normal cardiac rhythm on an electrocardiogram.

Diffusion-weighted brain magnetic resonance imaging (MRI) showed several scattered high signal intensities in the subcortical white matter of right frontal and parietal lobes, consistent with cortical border-zone area (between right anterior cerebral artery and MCA and between right MCA and posterior cerebral artery) (Figure 1). Brain magnetic resonance angiography showed a focal severe stenosis at right proximal M1 segment and mild stenosis at left proximal M1 segment (Figure 1).

She was treated with antiplatelet agents, atorvastatin and hydration with normal saline and clonazepam for chorea. Her symptoms improved noticeably from the very next day.

Hemichorea is usually caused by brain lesions involving basal ganglia structures, drugs, infection of central nervous system, autoimmune diseases, genetic causes, or metabolic causes [4]. The precise

Copyright (C) 2022 Korean Society of Geriatric Neurology

This is an Open Access article distributed under the terms of the Creative Commons Attribution Non-Commercial License (http://creativecommons.org/licenses/by-nc/4.0/) which permits unrestricted non-commercial use, distribution, and reproduction in any medium, provided the original work is properly cited. 

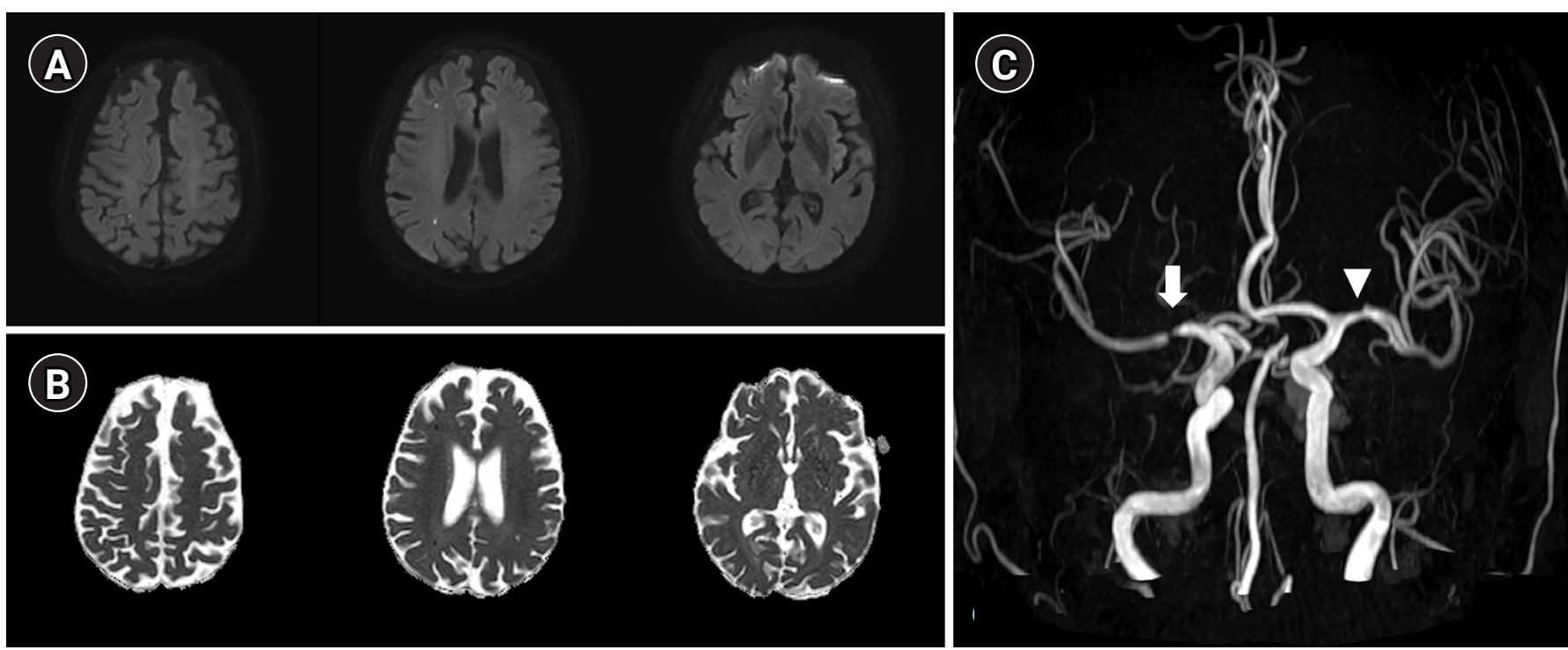

Figure 1. (A) Diffusion-weighted and (B) apparent diffusion coefficient magnetic resonance (MR) images demonstrate acute small infarctions in the border zone between right anterior cerebral artery and middle cerebral artery (MCA) and between right MCA and posterior cerebral artery. MR angiography (C) demonstrates a severe stenosis of the right MCA (arrow) and mild stenosis of the left MCA (arrowhead).

mechanism of post-stroke hemichorea remains uncertain [1]. It is believed to be due to ischemic damage in part of the motor circuit including basal ganglia or thalamus, resulting in impaired motor control.

However, as several cases of hemichorea have been reported in stroke of cortical lesions, other mechanisms have been suggested. Reduced cortical input signals cause thalamic inhibition through subtalamic nucleus, resulting in hemichorea [5]. Additionally, sensory loss through decreased sensory input to the motor cortex may also play a role, as seen with thalamic or parietal cortical lesions [6].

On the other hand, in our case, hemichorea occurred in a patient with stroke lesions only in the subcortical white matter of frontal and parietal lobes, sparing the basal ganglia structure and the brain cortex. Although mechanism for hemichorea in our patient remains unclear, it suggests another mechanism of hyperkinetic movement disorder which is different from previously known.

The subcortical white matter makes up about half of the volume of a normal human brain [7]. It contains short $U$ fibers and pyramidal dendrites, building neural connections between the cortex and the subcortical structures and forming the foundation of neural networks. This network makes up the foundation of neural networks for cognitive, sensory, motor and behavioral integration [8]. We presume that the ischemic insult of these structures would have disrupted the balance of motor network, although the lesion was very small.
Another hypothesis is that striatal hypoperfusion associated with severe atherosclerosis of right MCA causes functional changes in basal ganglia and results in reversible hemichorea. Previous reports demonstrated that hemichorea subsided after carotid artery stenting or carotid endarterectomy in patients with severe carotid artery stenosis [9]. However, although computed tomography perfusion imaging was not performed in our case, the fact that the symptoms persisted for more than 10 days and no additional lesions were observed even in subsequent follow-up brain MRI suggests that the ischemic insult was more likely to be limited to subcortical white matter.

Strokes causing involuntary movement disorders, while anatomically heterogeneous, localize to a common functional network [10]. We have demonstrated in this case that hyperkinetic movement disorders can appear in patients with stroke lesions only in the subcortical white matter of frontal and parietal lobes. Further studies will be needed on the functional role of the subcortical white matter of frontal and parietal lobes in the motor circuit.

\section{Notes}

\section{Conflicts of Interest}

The authors have no potential conflicts of interest to disclose.

\section{Funding}

None. 


\section{Author Contributions}

Conceptualization: SEA, PWC; Investigation: SEA, YHJ, SK; Methodology: SEA, PWC; Project administration: SEA; Writing-original draft: SEA; Writing-review \& editing: all authors.

\section{ORCID}

So-Ei Ann, https://orcid.org/0000-0003-2744-5651

Suho Ro, https://orcid.org/0000-0003-3378-561X

Yun Hyeong Jeong, https:// orcid.org/0000-0001-5277-3978

Sumin Kim, https://orcid.org/0000-0002-8523-883X

Pil-Wook Chung, https://orcid.org/0000-0002-8726-4812

\section{References}

1. Fukui T, Hasegawa Y, Seriyama S, Takeuchi T, Sugita K, Tsukagoshi H. Hemiballism-hemichorea induced by subcortical ischemia. Can J Neurol Sci 1993;20:324-328.

2. Ghika-Schmid F, Ghika J, Regli F, Bogousslavsky J. Hyperkinetic movement disorders during and after acute stroke: the Lausanne Stroke Registry. J Neurol Sci 1997;146:109-116.

3. Barinagarrementeria F, Vega F, DelBrutto OH. Acute hemichorea due to infarction in the corona radiata. J Neurol 1989; 236:371-372.
4. Damani A, Ghoshal A, Salins N, Deodhar J, Muckaden MA. Management of hemichorea hemiballismus syndrome in an acute palliative care setting. Indian J Palliat Care 2015;21:7275.

5. Nambu A. A new dynamic model of the cortico-basal ganglia loop. Prog Brain Res 2004;143:461-466.

6. Carbayo A, Sarto J, Santana D, Compta Y, Urra X. Hemichorea as presentation of acute cortical ischemic stroke. Case series and review of the literature. J Stroke Cerebrovasc Dis 2020;29: 105150 .

7. Engelhardt E, Moreira DM, Laks J. The brain subcortical white matter and aging: a quantitative fractional anisotropy analysis. Dement Neuropsychol 2009;3:228-233.

8. Xie S, Zhang Z, Chang F, Wang Y, Zhang Z, Zhou Z, et al. Subcortical white matter changes with normal aging detected by multi-shot high resolution diffusion tensor imaging. PLoS One 2016;11:e0157533.

9. Kim DW, Ko Y, Jang SH, Yoon SJ, Oh GS, Lee SJ, et al. Acute hemichorea as an unusual presentation of internal carotid artery stenosis. J Mov Disord 2013;6:17-20.

10. Laganiere S, Boes AD, Fox MD. Network localization of hemichorea-hemiballismus. Neurology 2016;86:2187-2195. 